\title{
Supporting Information: Electron-Induced Spin-Crossover in Self-Assembled Tetramers
}

\author{
Sven Johannsen, ${ }^{\dagger}$ Sascha Ossinger, ${ }^{\ddagger}$ Troels Markussen, $₫$ Felix Tuczek, ${ }^{\ddagger}$ \\ Manuel Gruber, ${ }^{*}, ;$ and Richard Berndt ${ }^{*, \dagger}$ \\ $\dagger$ Institut für Experimentelle und Angewandte Physik, Christian-Albrechts-Universität, \\ 24098 Kiel, Germany \\ $\ddagger$ Institut für Anorganische Chemie, Christian-Albrechts-Universität, 24098 Kiel, Germany \\ ฯSynopsys Denmark, Fruebjergvej 3, Postbox 4, DK-2100 Copenhagen, Denmark \\ $\S$ Faculty of Physics, University of Duisburg-Essen, 47057 Duisburg, Germany \\ E-mail: manuel.gruber@uni-due.de; berndt@physik.uni-kiel.de
}

\section{$\mathrm{Zn}\left(\mathrm{H}_{2} \mathbf{B}(\mathbf{p z})(\mathrm{pypz})\right)_{2}$ complexes on $\operatorname{Ag}(111)$}

As pointed out in the main manuscript, we verified the SCO nature of the switching by performing similar measurements on a complex with an identical geometry but different metal ion ( $\mathrm{Zn}$ instead of $\mathrm{Fe}$ ). The $3 d$ orbitals of the $\mathrm{Zn}^{2+}$ ion are fully occupied such that no $\mathrm{SCO}$ is expected. Upon deposition of the $\mathrm{Zn}$ compound on $\mathrm{Ag}(111)$, we observe the self-assembly of tetramers similar to those formed by the Fe compound (Figure S1). Nonetheless, as may be expected from a larger occupation of the antibonding $e_{g}$ orbitals, the $\mathrm{Zn}$ complexes are more fragile and a significant number of fragments is observed on $\mathrm{Ag}(111)$. One such fragment appears as a protrusion next to the top right molecule of the cluster (Figure S1).

We performed more than 160 switching attempts per molecule with tunneling parameters that would have lead to switching of $\mathrm{Fe}\left(\mathrm{H}_{2} \mathrm{~B}(\mathrm{pz})(\mathrm{pypz})\right)_{2}$ with high success rates. However, none of the attempts was successful. In particular, we did not observe any telegraph noise in time series, nor noticeable changes of the STM topographs. Incremental increase of the control voltage up to values above $-3.5 \mathrm{~V}(-400 \mathrm{pA})$ did not induce switching of a complex but rather lead to the destruction of the investigated tetramer.

These observations show the importance of the central metal ion for the switching and suggest that the switching of $\mathrm{Fe}\left(\mathrm{H}_{2} \mathrm{~B}(\mathrm{pz})(\mathrm{pypz})\right)_{2}$ is due to $\mathrm{SCO}$.

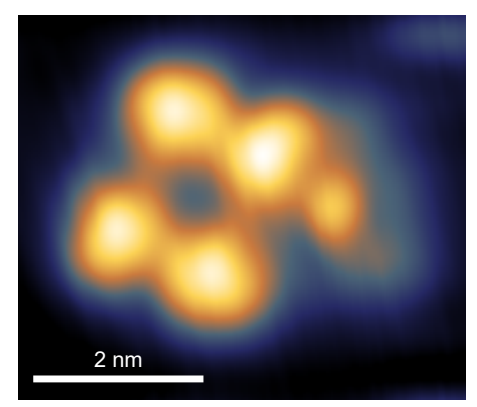

Figure S1: Topograph $(0.5 \mathrm{~V}, 10 \mathrm{pA})$ of a $\mathrm{Zn}\left(\mathrm{H}_{2} \mathrm{~B}(\mathrm{pz})(\mathrm{pypz})\right)_{2}$ tetramer on $\mathrm{Ag}(111)$. An additional protrusion on the right side is attributed to a fragment.

\section{Switching of single $\mathbf{F e}\left(\mathrm{H}_{2} \mathbf{B}(\mathrm{pz})(\mathrm{pypz})\right)_{2}$ complexes}

The impossibility to switch two of the molecules of a tetramer reflects steric hindrance in the tetramers. This geometric constraint is removed in dimers and switching is indeed observed as discussed in the manuscript. To further test the relevance of geometric constraints, we investigated single molecules at the border of tetramers (Figure S2). Such "pentamer" structures are observed on the pristine sample. The additional molecule next to the tetramer is not affected by the geometrical constraints, and switching between a low and high state is indeed possible (Figure S2).

The bonding geometry of the fifth molecule to the tetramer is not known in detail. Nonetheless, in most of the cases, we observed that the fifth molecule appears to be attracted between the pyrazole end groups 
of a molecule in the tetramer (corner of a tetramer). Two kinds of intermolecular interactions may therefore be involved: a $\pi-\pi$ interaction and electric dipole-dipole interaction. Gas-phase DFT calculations reveal a dipole moment of the compound of approximately 7 Debye. From simple point dipole considerations, an intermolecular interaction energy on the order of tens of millielectronvolts is expected, which would be sufficient to stabilize such structures at low temperatures.
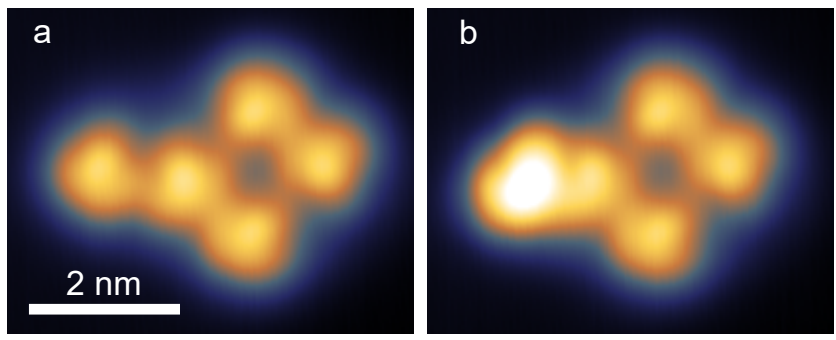

Figure S2: Topographs of a $\mathrm{Fe}\left(\mathrm{H}_{2} \mathrm{~B}(\mathrm{pz})(\mathrm{pypz})\right)_{2}$ attached to a tetramer. An additional protrusion on the left side of the tetramer, identified as a individual Fe complex in his a pristine and $\mathbf{b}$ switched state. The conversion between these states was induced by placing the tip above the molecule at a sample voltage of $1.2 \mathrm{~V}$ at $5 \mathrm{pA}$. Imaging conditions: $0.5 \mathrm{~V}, 5 \mathrm{pA}$.

\section{Structure of the gas-phase molecule}

Figure $\mathrm{S} 3$ shows structures of $\mathrm{Fe}\left(\mathrm{H}_{2} \mathrm{~B}(\mathrm{pz})(\mathrm{pypz})\right)_{2}$ in the LS and HS states resulting from gas-phase calculations. In the LS state, a pyrazole moiety of each ligand is tilted relative to the ligand plane (see for instance the angle of $10^{\circ}$ marked in Figure S3 a). Upon SCO, the tilt angle is significantly increased $\left(35^{\circ}\right.$, Figure S3 b). As SCO involves a change of the pyrazole tilt angles, no SCO is expected when steric hindrance blocks the tilting of the pyrazole.
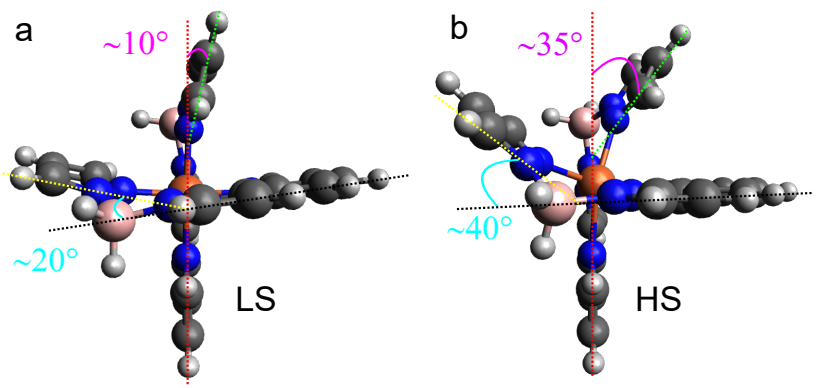

Figure S3: Calculated gas-phase structure of $\mathrm{Fe}\left(\mathrm{H}_{2} \mathrm{~B}(\mathrm{pz})(\mathrm{pypz})\right)_{2}$ in $\mathbf{a}$ the LS and $\mathbf{b}$ the HS state. The angle between the pyrazole moieties and the ligand plane indicated in magenta (cyan) is $\approx 10^{\circ}\left(\approx 20^{\circ}\right)$ and $\approx 35^{\circ}\left(\approx 40^{\circ}\right)$ in the LS and HS states, respectively. Note that only one of the two enantiomers is shown.

\section{Tetramer simulations}

In addition to the gas-phase simulations of monomers, we have performed calculations of tetramers in the gas-phase and adsorbed on $\mathrm{Ag}(111)$ using QuantumATK. ${ }^{\mathrm{S} 1, \mathrm{~S} 2}$ The atomic structure with a tetramer on four layers of $\mathrm{Ag}(111)$ contains 548 atoms, which is computationally very demanding with the B3LYP hybrid functional. For this reason, we performed the structural relaxations of tetramers in the gas phase and on $\operatorname{Ag}(111)$ with the generalized gradient approximation (GGA) for the exchange-correlation functional. We used the $\mathrm{PBE}^{\mathrm{S} 3}$ functional with an additional Hubbard$\mathrm{U}$ parameter of $4.0 \mathrm{eV}$ on the Fe $d$-orbitals. Furthermore, we included van-der-Waals interactions via the Grimme DFT-D3 correction to the total energy. ${ }^{\mathrm{S}}$ For the structural relaxation we employed a DoubleZetaPolarized basis set.

\section{Gas-phase Tetramer}

We verified that the relaxed structures of the monomers in the LS and HS configurations calculated with $\mathrm{PBE}+\mathrm{U}$ closely agreed with the structures obtained with B3LYP as described in the previous section.

Next, we proceeded to finding the structure of the tetramers. The relaxation of the tetramers was done in two steps. We started from the relaxed monomer structures and fixed all internal coordinates of each monomer and relaxed the structure by rigidly moving the monomers to the minimum energy position. In the second step, a full relaxation of all atomic coordinates was carried out until the maximum force was below $0.05 \mathrm{eV} / \AA$. The relaxed structure is shown in Figure S4. The calculated binding energy of the tetramer with four LS monomers is $3.91 \mathrm{eV}$. The distances shown in the figure are measured between the topmost hydrogen atoms in each monomer. The rectangular shape of the optimized tetramer matches the STM topographs shown in the main manuscript. Within one 'dimer' of the tetramer consisting of a 'A' and 'B' enantiomers indicated in Figure $\mathrm{S} 4$ we observe a slight difference in one of the angles between the pyrazole moieties and the ligand plane. In enantiomer B in Figure $\mathrm{S} 4$ the angle of $\sim 15^{\circ}$ is slightly larger than the corresponding angle of $\sim 10^{\circ}$ in enantiomer A.

The different angles in enantiomers A and B (Figure S4) already suggest that enantiomer B may be easier to switch to a HS state, where the angles are larger. To further address the switching capability of the monomers we calculated the gas-phase structure of tetramers in which we replaced one of the LS monomers A or B with a HS monomer and subsequently relaxed the atomic coordinates. The calculated total energy of the structure with the HS monomer in enantiomer B turned out $0.27 \mathrm{eV}$ lower that the tetramer with enantiomer $\mathrm{A}$ in a HS state. This energy difference supports the model presented in Figure 5 of the main manuscript and indicates that one of the enantiomers (A) is unable to switch due to steric hindrance. 


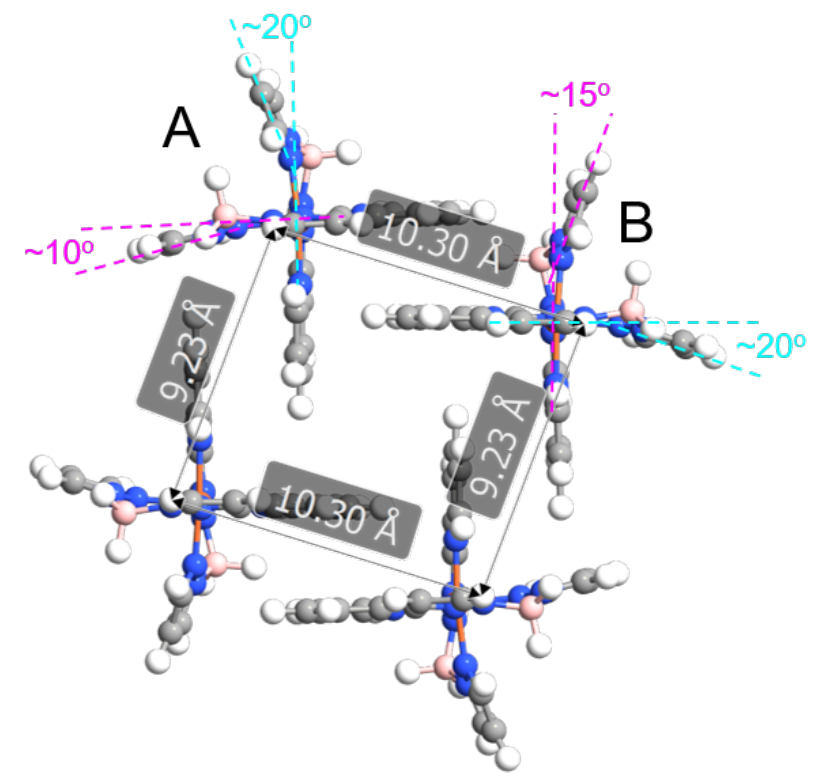

Figure S4: Calculated gas-phase structure of a tetramer with four LS monomers. The indicated distances are measured between the topmost hydrogen atoms of each monomer. The formation of a rectangular shape is in agreement with the STM topographs.

\section{Tetramer on $\operatorname{Ag}(111)$}

Figure S5 shows the relaxed geometry of a tetramer with four LS monomers adsorbed on four layers of $\mathrm{Ag}(111)$. For the structural relaxation, we fixed the three bottom Ag layers while doing a full geometry optimization of the remaining atoms. The $k$-point sampling was $(2,2,1)$, the real-space grid was determined by a density mesh cutoff of 80 Hartree, and we used a DoubleZetaPolarized basis set.

The calculated binding distance is $2.02 \AA$ as indicated in a. In qualitative agreement with the STM topographs we find that the monomers are arranged in a rectangular array with distances between the topmost hydrogen atoms of $9.23 \AA$ and $10.03 \AA$ as indicated in $\mathbf{b}$.

We next substituted one of the LS monomer with the HS configuration and repeated the structural relaxation using the $\mathrm{PBE}+\mathrm{U}$ functional. Subsequently, we performed a calculation with the B3LYP exchange correlation functional using a SingleZetaPolarized basis set.

Figure S6 shows the projected density of states (PDOS) on the HS monomer and neighboring LS monomer. The PDOS of monomer $\mathrm{A}$ is defined as

$$
D_{A}(E)=\sum_{i} \sum_{\mu \in A} w_{i}\left|\left\langle\phi_{\mu} \mid \psi_{i}\right\rangle\right| \delta\left(E-\epsilon_{i}\right)
$$

where the sum over $i$ runs over all eigenstates $\left|\psi_{i}\right\rangle$ in the system with eigenvalues $\epsilon_{i}$ including $k$-point summation and corresponding weight factors $w_{i}$. The $\mu$ sum runs over basis orbitals centered on atoms belonging to monomer $\mathrm{A}$. In the calculations, the $\delta$-function

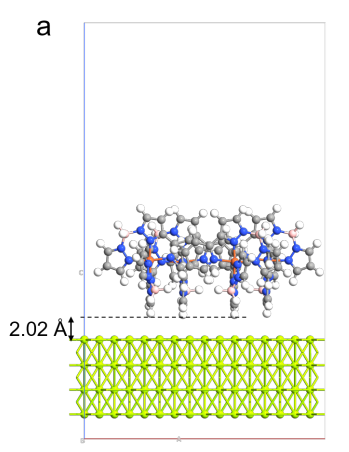

b

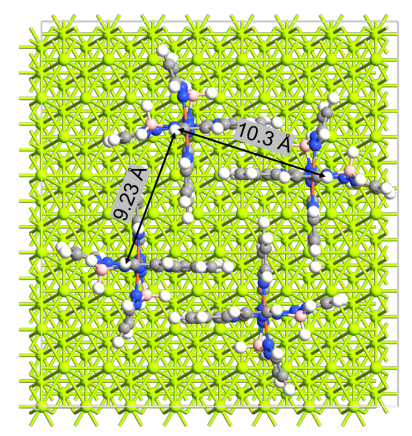

Figure S5: Calculated adsorption geometry of four LS monomers on four layers of $\mathrm{Ag}(111)$. The binding distance is $2.02 \AA$ as indicated in a. The distance between the topmost hydrogen atoms on each monomer is indicated in $\mathbf{b}$.

is replaced by a Gaussian with a width of $0.025 \mathrm{eV}$. The PDOS of monomer B is easily obtained by replacing A by B. It is evident from Figure $\mathrm{S} 6$ that the HS monomer has a smaller HOMO-LUOMO gap of $2.15 \mathrm{eV}$ than the LS monomer with $2.62 \mathrm{eV}$. The smaller gap of the HS monomer is in accordance with the gas-phase results, where we find HOMO-LUMO gaps of $2.26 \mathrm{eV}$ and $2.73 \mathrm{eV}$ for the HS and LS monomers, respectively, using the same computational settings. The smaller HOMOLUMO gap and, in particular, the reduced LUMO energy of the HS monomer is consistent with the larger apparent height in STM topographs presented in the main manuscript.

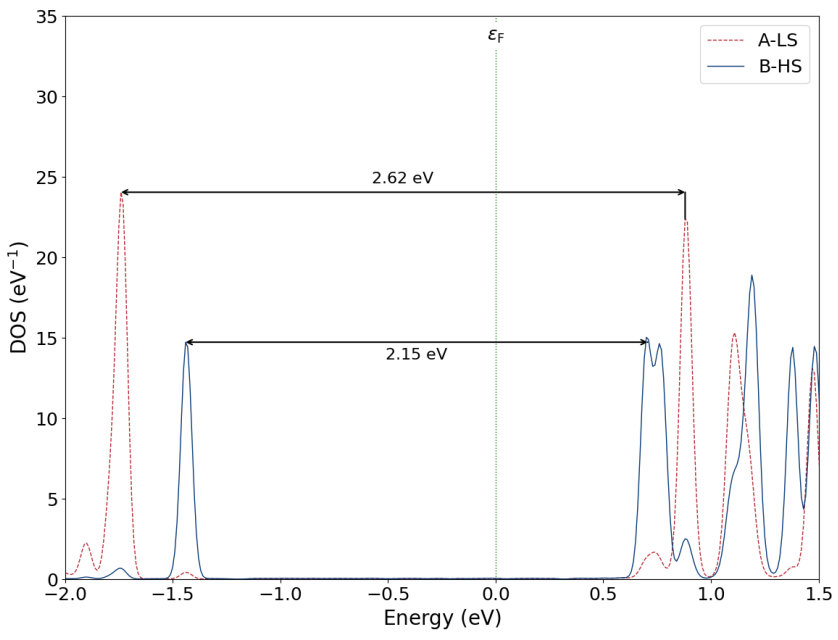

Figure S6: Projected density of states (PDOS) of the tetramer with three LS and one HS monomer on $\mathrm{Ag}(111)$ also shown in Figure S7. The density of states is projected onto the basis orbitals located on the HS monomer and on the neighboring LS monomer. The LUMO orbital on the HS is $0.18 \mathrm{eV}$ lower in energy than the LUMO peak of the LS monomer.

To further address the apparent height difference observed in the experiments we also calculated and ana- 
lyzed the partial electron density. The partial electron density is obtained from eigenstates within a specified energy interval $\left[E_{0} ; E_{1}\right]$ and is defined as

$$
n_{E_{0}, E_{1}}(\mathbf{r})=\sum_{i} w_{i}\left|\left\langle\mathbf{r} \mid \psi_{i}\right\rangle\right| \Theta\left(\epsilon_{i}-E_{0}\right) \Theta\left(E_{1}-\epsilon_{i}\right),
$$

where the $i$-sum again runs over both $k$-points and eigenstates. $\Theta(x)$ is the Heaviside step function. Within the Tersoff-Hamann approximation, the current measured by the STM at a sample voltage of $V$ is proportional to the partial electron density $n_{0, e \cdot V}(\mathbf{r})$. Figure $\mathrm{S} 7$ shows the partial electron density at a constant height of $2.12 \AA$ above the topmost hydrogen atoms. The calculated image shows a bright spot above the HS monomer as compared to the LS monomers. Although this is not a direct comparison to the STM measurements, which show the height variations at a constant current, the simulated STM image together with the PDOS plot in Figure S6 indicates that the taller molecules observed in the measured STM topographs correspond to the HS state.

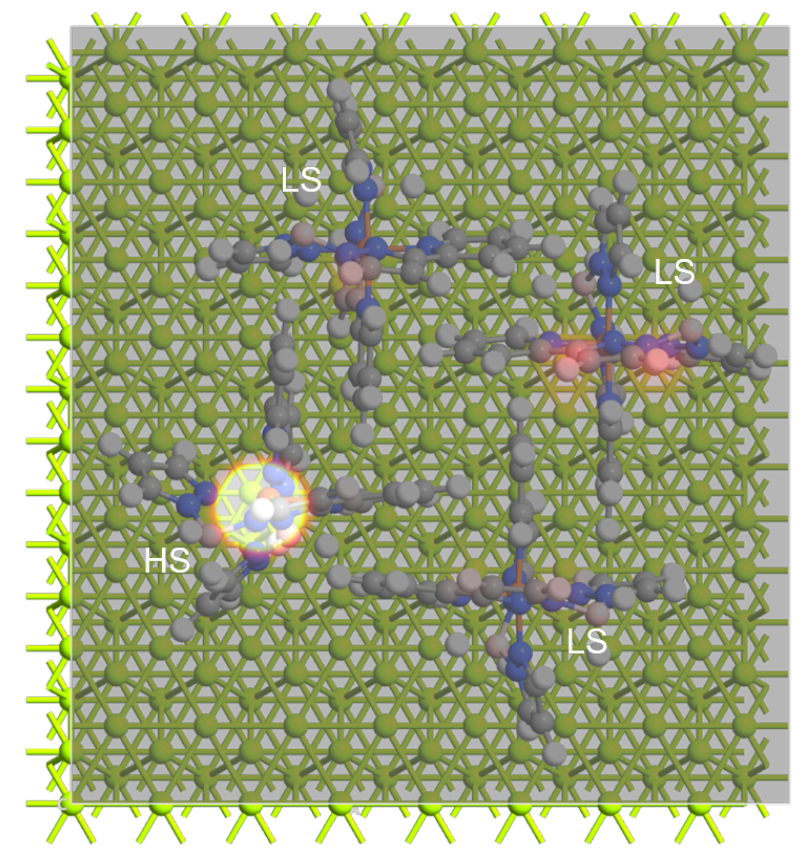

Figure S7: Simulated constant-height STM image of a tetramer with three LS and one HS monomer on $\operatorname{Ag}(111)$. The figure shows the partial density summed in the energy interval $[0,0.65] \mathrm{eV}$ for a constant height $2.12 \AA$ above the topmost hydrogen atoms. Yellowish area at the HS monomer indicate a significant higher density than on the rest of the supercell. A sticks and balls representation of the supercell is overlaid.

\section{Simple model of electronic cou- pling between LUMO orbitals}

We now describe a simple model to address the remote switching and, in particular, the different switching efficiency from LS to HS and vice versa.

We consider a transition of an electron initially in the LUMO orbital located on a non-switchable monomer (A) to the LUMO of the neighboring, switchable monomer (B). The initial LUMO state is always in a LS configuration with the energy $E_{L U M O}^{L S}$, while the final state is a LS LUMO for LS $\rightarrow$ HS switching and a HS LUMO states for HS $\rightarrow$ LS switching. We estimate the transition probabilities using Fermi's golden rule

$$
\Gamma_{A \rightarrow B}=\frac{2 \pi}{\hbar}\left|\left\langle\psi_{L S}^{(A)}\left|H^{\prime}\right| \psi_{L S / H S}^{(B)}\right\rangle\right|^{2} \rho_{L S / H S}^{(B)}\left(E_{L S}^{A}\right)
$$

where $\left|\psi_{L S}^{(A)}\right\rangle$ is the LUMO orbital located on the LS monomer $\mathrm{A},\left|\psi_{L S / H S}^{(B)}\right\rangle$ is the LUMO orbital on monomer $\mathrm{B}$, which can either be in a LS or HS configuration. The perturbation to the Hamiltonian $H^{\prime}$ is detailed below. Finally $\rho_{L S / H S}^{(B)}\left(E_{L S}^{A}\right)$ is the density of states on the B monomer (either LS or HS) but always evaluated at the LUMO energy for the LS monomer A. From the projected density of states shown in Figure $\mathrm{S} 6$ we estimate the LUMO peak separation to be $\Delta E \approx 0.2 \mathrm{eV}$ and the broadening of the peaks to be $\gamma \approx 0.1 \mathrm{eV}$. Next, a Lorentzian shape is assumed for the LUMO density of states such that

$$
\rho_{L S}^{(B)}\left(E_{L S}^{A}\right) \propto \frac{\gamma}{\left(E_{L S}^{B}-E_{L S}^{A}\right)^{2}+\gamma^{2}}=\frac{1}{\gamma}
$$

since $E_{L S}^{B}=E_{L S}^{A}$ and

$$
\rho_{H S}^{(B)}\left(E_{L S}^{A}\right) \propto \frac{\gamma}{\left(E_{H S}^{B}-E_{L S}^{A}\right)^{2}+\gamma^{2}}=\frac{\gamma}{(\Delta E)^{2}+\gamma^{2}}
$$

The coupling matrix elements entering Eq. 3 are estimated as follows. To simplify the notation, we consider a dimer although the final results are obtained for tetramers. We obtain the full Hamiltonian matrix from the DFT calculation with elements $\mathbf{H}_{i j}=\left\langle\phi_{i}^{\mu}|\hat{H}| \phi_{j}^{\nu}\right\rangle$, where $\left|\phi_{i}^{\mu}\right\rangle$ is an atomic basis orbital located on atom $\mu$ and $\hat{H}$ is the Hamiltonian operator from the converged DFT calculation.

We divide the total Hamiltonian matrix for a given spin (either up or down) into a two-by-two form:

$$
\mathbf{H}=\left(\begin{array}{cc}
\mathbf{H}_{1} & \mathbf{0} \\
\mathbf{0} & \mathbf{H}_{2}
\end{array}\right)+\left(\begin{array}{cc}
\mathbf{0} & \mathbf{H}_{12} \\
\mathbf{H}_{21} & \mathbf{0}
\end{array}\right)
$$

and similarly for the overlap matrix. The sub-matrix $\mathbf{H}_{1}$ contains matrix elements between atomic basis orbitals located on monomer 1 and likewise for the submatrix $\mathbf{H}_{2}$. The second term in Eq. 6 contains matrix elements where one basis orbital is located on monomer 1 and the other basis orbital on monomer 2. This matrix thus describes the coupling between the monomers 
and we will treat this as the perturbation $H^{\prime}$.

We now find the eigenvalues and eigenvectors for the monomer subsystems 1 and 2, i.e. we solve the equation

$$
\mathbf{H}_{1} \psi_{1}^{(n)}=\epsilon^{(n)} \mathbf{S}_{1} \psi_{1}^{(n)}
$$

for the $n$ 'th eigenstate of monomer 1 and similarly for monomer $2 . \quad \mathbf{S}_{1}$ is the basis set overlap matrix for monomer 1 , where the matrix element $i j$ reads $\left(S_{1}\right)_{i j}=\left\langle\phi_{i} \mid \phi_{j}\right\rangle$. The coupling between the LUMO orbitals of monomer 1 and monomer 2 is then calculated as

$$
\Gamma_{12}=\left(\begin{array}{c}
\psi_{1}^{L U M O} \\
\mathbf{0}
\end{array}\right)^{\dagger} \mathbf{H}^{\prime}\left(\begin{array}{c}
\mathbf{0} \\
\psi_{2}^{L U M O}
\end{array}\right)
$$

Using a gas-phase tetramer with four LS monomers we calculate the coupling $\left|\left\langle\psi_{L S}^{A}\left|H^{\prime}\right| \psi_{L S}^{B}\right\rangle\right|$. Similarly, from a tetramer with a single HS monomer and three LS monomers we obtain $\left|\left\langle\psi_{L S}^{A}\left|H^{\prime}\right| \psi_{H S}^{B}\right\rangle\right|$.

We can now estimate the ratio of the transition rates $\Gamma_{(A, L S) \rightarrow(B, L S)}$ for the remote $L S \rightarrow H S$ switching event and $\Gamma_{(A, L S) \rightarrow(B, H S)}$ for the remote $H S \rightarrow L S$ switching event as

$$
\frac{L S \rightarrow H S}{H S \rightarrow L S}=\frac{\left|\left\langle\psi_{L S}^{A}\left|H^{\prime}\right| \psi_{L S}^{B}\right\rangle\right|^{2}}{\left|\left\langle\psi_{L S}^{A}\left|H^{\prime}\right| \psi_{H S}^{B}\right\rangle\right|^{2}} \frac{(\Delta E)^{2}+\gamma^{2}}{\gamma^{2}}
$$

The results from both $\mathrm{PBE}+\mathrm{U}$ and B3LYP calculatitons are summarized in Table S1. The estimated experimental ratio is obtained from the multiplying factors of 30 and 130 for remote $L S \rightarrow H S$ and $H S \rightarrow L S$ switching, respectively from Figure 8 in the main manuscript. From this we estimate the ratio as $(L S \rightarrow H S) /(H S \rightarrow$ $L S)=130 / 30=4.3$. The ratios determined from the DFT calculations strongly depend on the used functional (9.0 with PBE+U and 47.1 with B3LYP), which implies a large uncertainty. Nonetheless, the ratios inferred from theory have a similar order of magnitude as the experimental one and support an intermolecular electronic transfer mechanism for the remote switching.

Table S1: Coupling elements between LUMO orbitals and estimated ratios of the transition rates involved in remote $L S \rightarrow H S$ and $H S \rightarrow L S$ switching. The experimental ratio is obtained from the multiplying factors of 30 and 130 from Figure 8 in the main manuscript.

\begin{tabular}{|c|c|c|c|}
\hline & PBE+U & B3LYP & Exp. \\
\hline$\left|\left\langle\psi_{L S}^{A}\left|H^{\prime}\right| \psi_{L S}^{B}\right\rangle\right|$ & $32 \mathrm{meV}$ & $20 \mathrm{meV}$ & - \\
\hline$\left|\left\langle\psi_{L S}^{A}\left|H^{\prime}\right| \psi_{H S}^{B}\right\rangle\right|$ & $22 \mathrm{meV}$ & $6 \mathrm{meV}$ & - \\
\hline$\frac{L S \rightarrow H S}{H S \rightarrow L S}$ & 9.0 & 47.1 & 4.3 \\
\hline
\end{tabular}

\section{Proportionality of rate and cur- rent}

Figure S8 shows the same data as in Figure 3 of the main text but with a linear scale to highlight the proportion- ality of the rates to the current. This proportionality indicates that the switching is a single electron process.
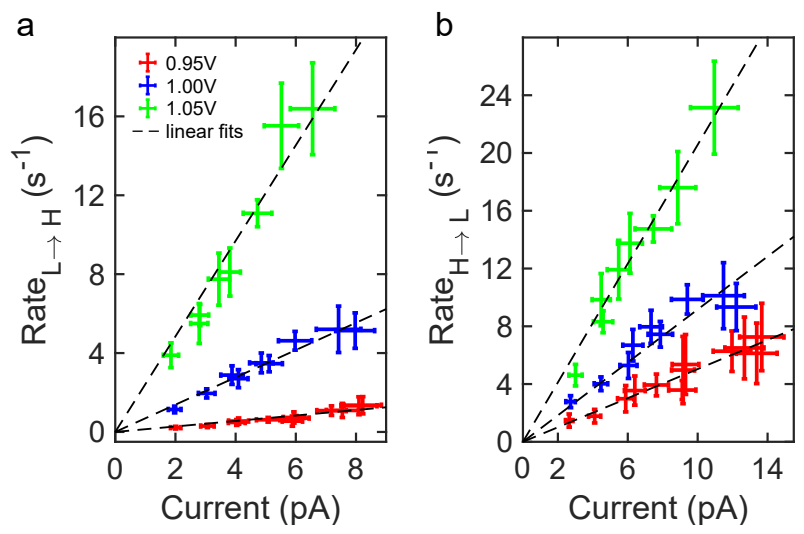

Figure S8: Current dependence of the switching rates. a Low-to-high and $\mathbf{b}$ high-to-low switching rates as a function of the tunneling current for different voltages (crosses). The dashed lines are a linear fit to the data. See caption to Figure 3 for further experimental details.

\section{Switching at negative voltages}

In the main manuscript, we considered switching at positive sample voltages, i. e. electron current from the tip to the molecule. Switching between the LS and HS state may also be realized at negative voltages as illustrated in Figure S9. However, switching at negative voltages appears to be more complex than at positive voltage. In particular, a detailed monitoring of the switching at negative voltages (not shown) suggests that an intermediate state is involved in the switching at this polarity. A detailed description of this intermediate state, and hence of the switching at negative sample voltages, is out of the scope of the present manuscript.

\section{Analysis of current time series}

The probability of a molecule to switch (under appropriate tunneling parameters) is expected to be independent of the history of the molecule. Consequently, the number of switching events within a time interval may be described by a Poisson distribution. We define the discrete random variable $\mathcal{X}$ as the number of switching events per time interval $\tau$. $\mathcal{X}$ follows a Poisson distribution:

$$
\mathcal{P}(\mathcal{X}=k)=\frac{\mu_{\tau}^{k} e^{-\mu_{\tau}}}{k !}
$$

where $k$ is a given realization of $\mathcal{X}$ and $\mu_{\tau}=\langle\mathcal{X}\rangle$ is the average number of switching events during $\tau$. The standard deviation of the distribution is given by $\sigma_{\mathcal{X}}=\sqrt{\langle\mathcal{X}\rangle}=\sqrt{\mu_{\tau}}$. The last property is useful for estimating the uncertainty of a measurement from the mean value. Below, we show that the switching events 


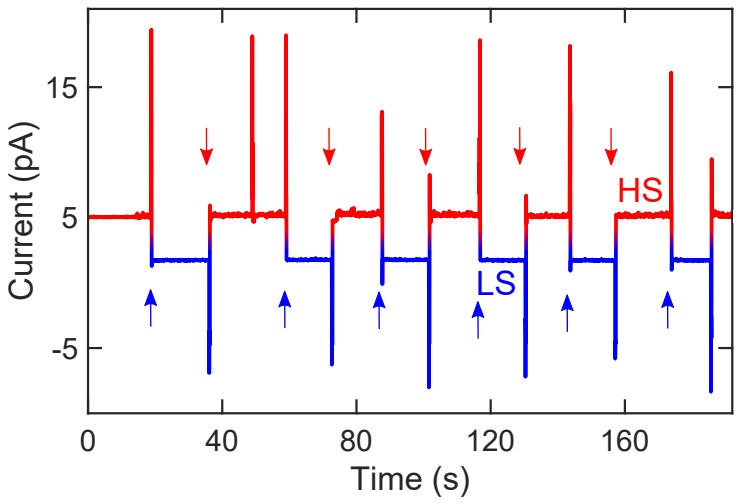

Figure S9: Switching with positive and negative voltage pulses. Evolution of the tunneling current across a switchable molecule for a constant tip-substrate distance (current feedback disabled). At the position indicated by the blue (red) arrows, a voltage pulse of $1 \mathrm{~V}$ $(-1 \mathrm{~V})$ was applied over $280 \mathrm{~ms}$. The current level in the absence of the pulses, measured with a DC voltage of $0.5 \mathrm{~V}$, is a readout of the molecular state.

in $\mathrm{Fe}\left(\mathrm{H}_{2} \mathrm{~B}(\mathrm{pz})(\mathrm{pypz})\right)_{2}$ on $\mathrm{Ag}(111)$ can indeed be described by a Poisson distribution. We then describe how the rates and related uncertainties were determined.

To test the distribution of the switching events we recorded long time series of the tunneling current, divided them into shorter intervals, counted the number of switching events, and compared the resulting histogram of the number of switching events per interval with the expectation from a Poisson distribution. We distinguished between low-to-high and high-to-low events because they are associated with different yields. This effectively leads to two separate time series, one with the molecule in its low state (referred to as time series low) and one with the molecule in its high state (referred to as time series high). This artificial separation is necessary because the molecule cannot exhibit a lowto-high switching when it already is in the high state. The time series low and high are divided into smaller intervals. The normalized histograms of the number of low-to-high and high-to-low switching in those intervals are shown in Figure S10 for a time series recorded at $1 \mathrm{~V}$ and a current of $5 \mathrm{pA}$ in the low state. In time intervals of $0.5 \mathrm{~s}$, we observe between 0 and 7 low-to-high switching events between 0 and 8 high-to-low processes. Poisson distributions (red) do indeed reproduce the histograms. We note that small deviations are visible $[e . g$. 4 high-to-low switching events (Figure S10 b) appear more often than predicted by the Poisson distribution] presumably owing to the limited size of the data set (total of 279 high-to-low switching in the present case).

For sufficiently long time series (low and high) the number of switching events provides an estimate of $\bar{\mu}_{\tau}$ with an associated uncertainty $\sqrt{\bar{\mu}_{\tau}}$. The switching rate $R$, defined as the average number of switching per second, is estimated from $R=\bar{\mu}_{\tau} / \tau$. The uncertainty of
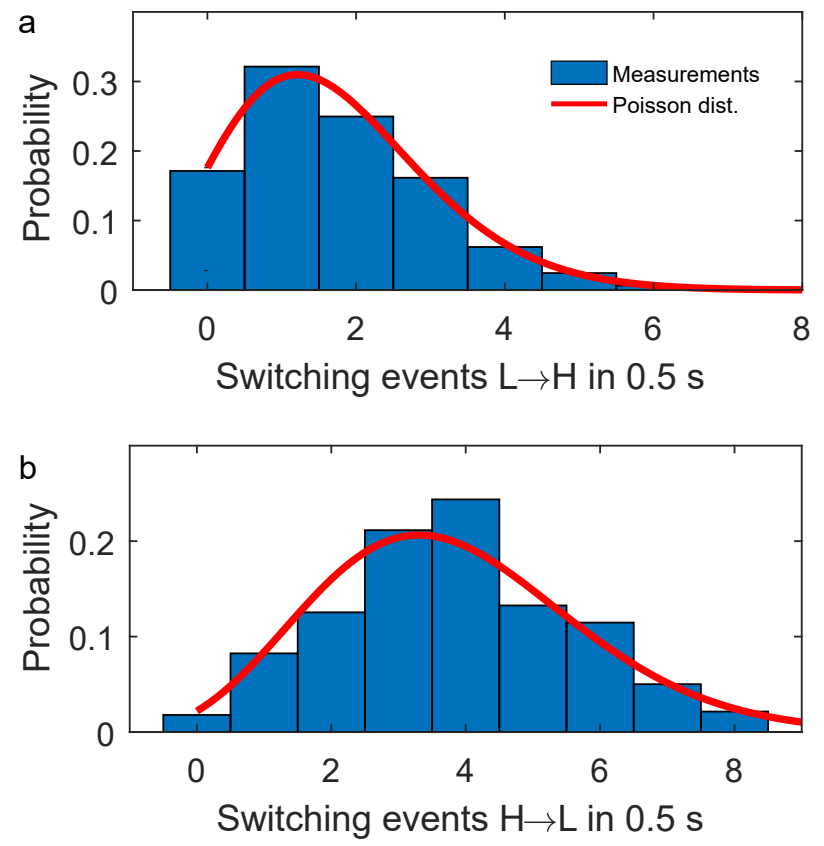

Figure S10: Number of switching events described by Poisson distribution. Histograms of the number of switching events in a time interval of $0.5 \mathrm{~s}$ (blue) a from the low to high state and $\mathbf{b}$ high to low state, along with Poisson distributions (red) with $\mathbf{a} \mu_{0.5 \mathrm{~s}}=1.74$ and $\mathbf{b}$ $\mu_{0.5 s}=3.82$. The factorial in Equation 10 has been replaced by $\int_{0}^{\infty} e^{-t} t^{k} d t$ for non-integer values of $k$.

the rate estimate is

$$
\sigma_{R}=\frac{\sqrt{\bar{\mu}_{\tau}}}{\tau}=\sqrt{\frac{R}{\tau}} .
$$

As expected a decrease of the uncertainty by a factor 2 requires a time interval that is four times longer.

The yield, describing the probability of a electron to induce switching, is inferred from

$$
Y=\frac{e R}{\langle I\rangle},
$$

where $e$ is the elementary charge and $\langle I\rangle$ the mean value of the current. We recall that we distinguish between low-to-high and high-to-low switching, which are linked to the mean current values in the low and high states, respectively. The uncertainty of the yield is related to those of the rate $\sigma_{R}$ and the current $\sigma_{I}$ as follows:

$$
\sigma_{Y}=\left(\frac{\sigma_{R}}{R}+\frac{\sigma_{I}}{\langle I\rangle}\right)|Y|
$$

\section{Spectroscopic data}

The spectroscopic data do unfortunately not exhibit any feature that would enable a direct and unambiguous identification of the spin states. Figure S11 shows $d I / d V$ spectra recorded over a large voltage range. Based on the yield versus voltage data, we may ex- 
pect an unoccupied molecular orbital at an energy of $\approx 1.5 \mathrm{eV}$. Such an orbital, however, is not resolved because the tetramers are not stable at voltages above $\approx 1 \mathrm{~V}$ and hop laterally. This motion induces abrupt changes in the differential conductance as shown in Figure S11.

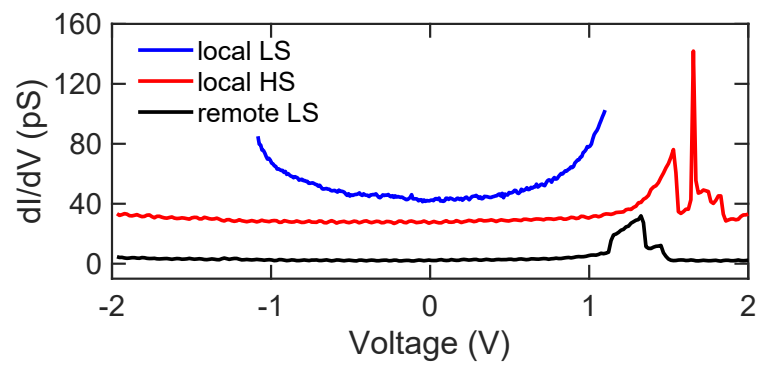

Figure S11: Differential-conductance spectra over a large voltage range. $d I / d V$ spectra acquired over a switchable molecule in the LS (blue) and in the HS (red) states and over a non-switchable neighboring molecule in the LS state (black). Besides strong variations of $d I / d V$ for voltages above $1 \mathrm{~V}$, which result from the lateral displacement of the investigated tetramer, the spectra are essentially free of features. The spectra were acquired using a lock-in amplifier with root-meansquare voltage modulations of $15 \mathrm{mV}(10 \mathrm{mV})$ for the red and black (blue) curves. Before disabling the current feedback, the tunneling conditions were $15 \mathrm{pA}$ and $-2 \mathrm{~V}(10 \mathrm{pA}$ and $-1 \mathrm{~V})$ for the red and black (blue) spectra. The red and blue spectra are shifted by $25 \mathrm{pS}$ and $40 \mathrm{pS}$, respectively.

Figure S12 shows $d I / d V$ spectra of a molecule in the HS (red) and LS (blue) states over a narrow range around the Fermi energy. No spin-related features such as a Kondo resonance or spin-flip excitation are apparent, which prevents a direct spin-state identification from the spectra. It should be noted that the absence of such features is not surprising. Spin-flip excitations have so far never been reported for SCO complexes and require magnetic anisotropy. Alternatively, a necessary condition for an observation of a Kondo resonance is a sufficiently strong electronic coupling between the spin of the molecule and the conduction electrons of the substrate. The induced lateral hopping of the tetramer, however, suggests that the molecule-substrate coupling is not particularly strong.

\section{Orientation of the tetramers}

The tetramers adopt six different orientations on the $\operatorname{Ag}(111)$ surface (Figure S13), which suggests a specific interaction between the molecules and the substrate. Considering the three equivalent close-packed directions of the $\mathrm{Ag}(111)$ surface, there are presumably two preferred adsorption geometries of the molecules on the surface.

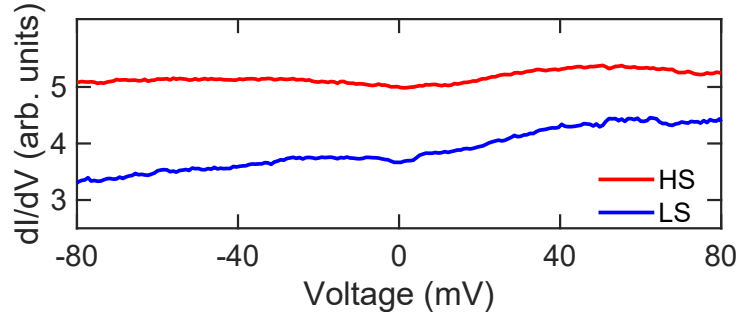

Figure S12: Differential-conductance spectra close to the Fermi energy. The $d I / d V$ spectra acquired on a switchable molecule in the HS (red) and LS (blue) states essentially appear featureless. The $d I / d V$ signal was recorded using a lock-in amplifier (root-meansquare voltage modulation of $2.5 \mathrm{mV}$ ) with the current feedback off (initial tunneling conditions of $80 \mathrm{pA}$ and $-100 \mathrm{mV}$ ). The red spectrum is vertically shifted for clarity.

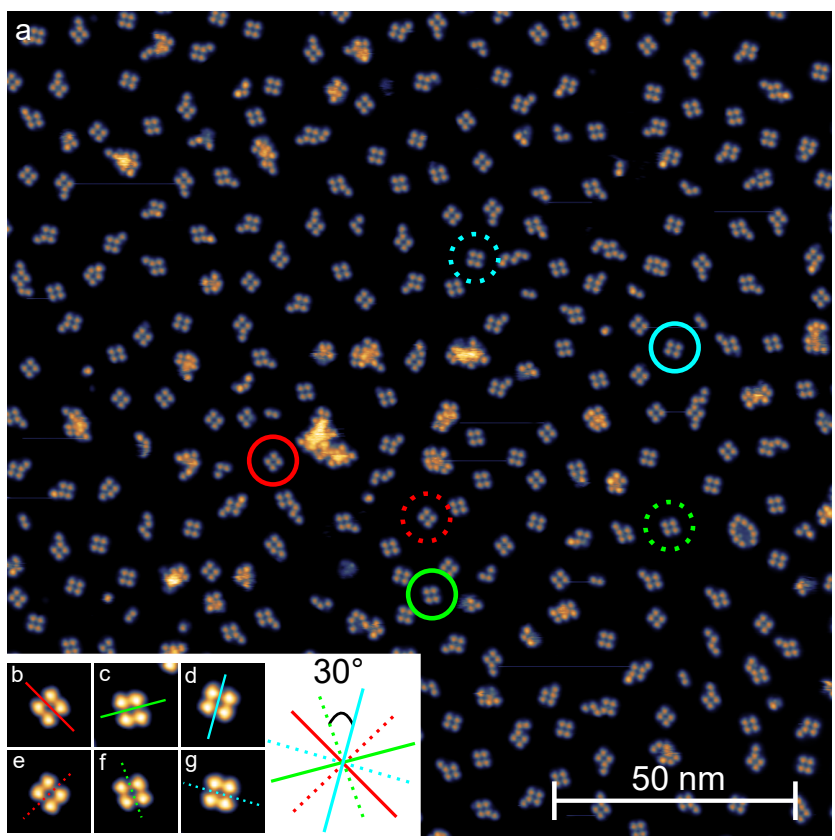

Figure S13: Orientations of the tetramers. a STM topograph of the Fe complex on $\mathrm{Ag}(111)$ showing a large number of tetramers along with few unidentified aggregates. $\mathbf{b}-\mathbf{g}$ Examples of the six different orientations of the tetramers observed on the surface. Tetramers may be seen as composed of two dimers. Lines are drawn between such dimers to highlight the different possible orientations. In this particular image, we counted 33, $25,38,38,36$ and 36 tetramers with orientations such as shown in $\mathbf{b}-\mathbf{g}$, respectively. Overall, the abundances of the the six orientations appear to be equal.

\section{Chirality of the tetramers}

The structure of the tetramers proposed in Figure 5a of the main text is chiral and one may expect the presence of a second enantiomer. Such an enantiomer is indeed observed (Figure S14). The topograph in Figure S14a 
cannot be reproduced by a mere rotation of that in Figure S14b, but requires a mirror symmetry operation.
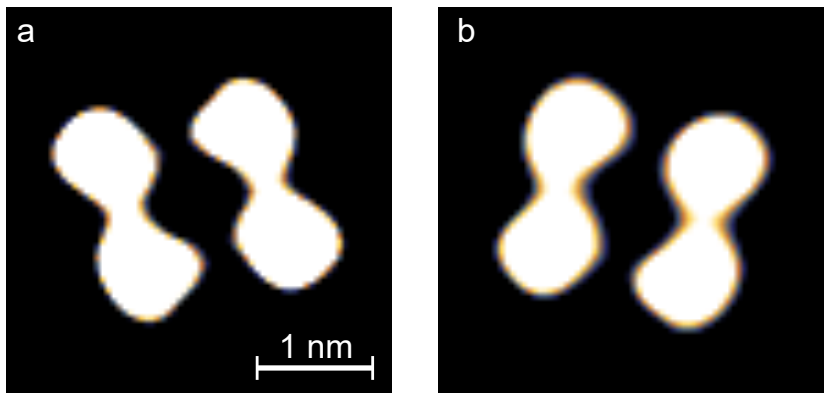

Figure S14: Chirality of the tetramers. Constantcurrent STM topographs of the two enantiomers found on the surface. The topograph $\mathbf{b}$ has been rotated by $30^{\circ}$ to simplify comparison. Note that the color scale has been saturated over a large fraction of the molecules to better visualize the chirality of the structure.

\section{References}

(S1) QuantumATK

2021.06,

version

S(www.synopsys.com/silicon/quantumatk.html), date of access: June 4, 2021.

(S2) Smidstrup, S.; Markussen, T.; Vancraeyveld, P.; Wellendorff, J.; Schneider, J.; Gunst, T.; Verstichel, B.; Stradi, D.; Khomyakov, P. A.; VejHansen, U. G.; Lee, M.-E.; Chill, S. T.; Rasmussen, F.; Penazzi, G.; Corsetti, F.; Ojanperä, A.; Jensen, K.; Palsgaard, M. L. N.; Martinez, U.; Blom, A. et al. QuantumATK: An Integrated Platform of Electronic and Atomic-Scale Modelling Tools. J. Phys. Condens. Matter 2019, 32, 015901.

(S3) Perdew, J. P.; Burke, K.; Ernzerhof, M. Generalized Gradient Approximation Made Simple. Phys. Rev. Lett. 1996, 77, 3865-3868.

(S4) Grimme, S.; Antony, J.; Ehrlich, S.; Krieg, H. A Consistent and Accurate $a b$ Initio Parametrization of Density Functional Dispersion Correction (DFT-D) for the 94 Elements H-Pu. J. Chem. Phys. 2010, 132, 154104. 


\section{Atomic coordinates}

Table S2: Atomic coordinates of relaxed structures inferred from gas-phase calculations of monomers.

\begin{tabular}{|c|c|c|c|c|c|c|c|c|c|c|c|c|}
\hline & \multicolumn{3}{|c|}{ Enantiomer A HS } & \multicolumn{3}{|c|}{ Enantiomer A LS } & \multicolumn{3}{|c|}{ Enantiomer B HS } & \multicolumn{3}{|c|}{ Enantiomer B LS } \\
\hline $\mathrm{Fe}$ & -0.481 & 4.685 & 4.116 & -0.118 & 4.610 & 3.949 & 4.969 & 0.398 & 10.671 & 4.605 & 0.473 & 10.838 \\
\hline $\mathrm{N}$ & 1.651 & 4.071 & 3.205 & 1.675 & 3.993 & 3.212 & 2.836 & 1.012 & 11.582 & 2.812 & & 11.575 \\
\hline $\mathrm{C}$ & 2.039 & 4.871 & 2.189 & 2.145 & 4.754 & 2.185 & 2.448 & 0.212 & 12.599 & 2.342 & 0.329 & 2.602 \\
\hline $\mathrm{C}$ & 3.151 & 4.554 & 1.394 & 3.319 & 4.417 & 1.500 & 1.336 & 0.529 & 13.393 & 1.168 & 0.666 & 13.287 \\
\hline $\mathrm{H}$ & 3.444 & 5.217 & 0.578 & 3.664 & 5.050 & 0.681 & 1.043 & -0.133 & 14.209 & 0.823 & 0.033 & 4.106 \\
\hline $\mathrm{C}$ & 3.859 & 3.384 & 1.660 & 4.018 & 3.273 & 1.8 & 0.628 & & 13.127 & 0.469 & & 2.911 \\
\hline $\mathrm{H}$ & 4.726 & 3.115 & 1.051 & 4.934 & 2.989 & 1.353 & -0.238 & 1.968 & 13.735 & -0.447 & 2.094 & 13.434 \\
\hline c & 3.440 & 2.557 & 2.706 & 3.525 & 2.493 & 2.926 & 1.047 & 2.526 & 12.081 & 0.962 & 2.590 & 11.861 \\
\hline $\mathrm{H}$ & 3.960 & 1.627 & 2.942 & 4.035 & 1.584 & 3.250 & 0.526 & 3.455 & 11.845 & 0.452 & 3.499 & 1.537 \\
\hline c & 2.325 & 2.947 & 3.451 & 2.350 & 2.891 & 3.562 & 2.162 & 2.136 & 11.336 & 2.137 & 2.192 & 1.225 \\
\hline $\mathrm{H}$ & 1.956 & 2.329 & 4.274 & 1.928 & 2.302 & 4.377 & 2.530 & 2.753 & 10.513 & 2.559 & 2.781 & 0.410 \\
\hline c & 1.213 & 6.059 & 1.976 & 1.312 & 5.908 & 1.889 & 3.274 & -0.975 & 12.811 & 3.175 & -0.824 & 2.898 \\
\hline $\mathrm{N}$ & 0.145 & 6.220 & 2.778 & 0.224 & 6.020 & 2.674 & 4.342 & -1.136 & 12.010 & 4.263 & -0.936 & 12.113 \\
\hline $\mathrm{N}$ & -0.472 & 7.352 & 2.432 & -0.461 & 7.099 & 2.309 & 4.960 & -2.268 & 12.355 & 4.949 & -2.015 & 12.478 \\
\hline C & 0.180 & 7.931 & 1.398 & 0.170 & 7.701 & 1.270 & 4.307 & -2.847 & 13.390 & 4.317 & -2.617 & 13.517 \\
\hline $\mathrm{H}$ & -0.175 & 8.867 & 0.975 & -0.235 & 8.609 & 0.832 & 4.663 & -3.783 & & 4.723 & -3.525 & 3.955 \\
\hline C & 1.278 & 7.137 & 1.067 & 1.319 & 6.9 & 0.9 & 3.209 & -2.053 & & & & 3.825 \\
\hline $\mathrm{H}$ & 2.009 & 7.318 & 0.284 & 2.043 & 7.183 & 0.179 & 2.478 & -2.234 & & 2.444 & -2.099 & 14.608 \\
\hline B & -1.644 & 7.914 & 3.305 & -1.623 & 7.635 & & 6.132 & -2.830 & & & & 11.579 \\
\hline $\mathrm{H}$ & -1.174 & 8.285 & 4.3 & -1.1 & & & 5.662 & -3.2 & & & & 10.659 \\
\hline $\mathrm{H}$ & -2.179 & 8.8 & 2.6 & -2.3 & & & 67 & -3 . & & & & 12.260 \\
\hline $\mathrm{N}$ & -2.691 & 6.7 & 7 & -2.4 & & & 179 & -1 . & & & & 1.009 \\
\hline $\mathrm{N}$ & -2.393 & 5.60 & 9 & -1.923 & 5.3 & & 31 & -0 & & & & 10.432 \\
\hline C & -3.544 & 4.9 & 5 & -2.934 & 4.6 & & 032 & & & & & 9.852 \\
\hline $\mathrm{H}$ & -3.534 & 3.9 & 4 & -2.756 & 3.7 & 5.422 & .022 & & & & & 9.365 \\
\hline C & -4.619 & 5.703 & 3.824 & -4.139 & 5.366 & 4.745 & 9.107 & -0.619 & & 627 & & 10.042 \\
\hline $\mathrm{H}$ & -5.675 & 5.445 & 3.814 & -5.132 & 5.086 & 5.087 & 10.163 & -0.361 & 10.973 & 9.620 & $-0 .($ & 9.700 \\
\hline C & -4.023 & 6.878 & 3.367 & -3.776 & 6.484 & 4.000 & 8.511 & -1.794 & 11.420 & 8.264 & -1.400 & 10.787 \\
\hline $\mathrm{H}$ & -4.457 & 7.772 & 2.926 & -4.368 & 7.315 & 3.624 & 8.945 & -2.688 & 11.861 & 8.856 & -2.231 & 11.163 \\
\hline $\mathrm{N}$ & 0.761 & 5.578 & 5.799 & 0.770 & 5.572 & 5.484 & 3.726 & -0.493 & 888 & .717 & -0.488 & 9.303 \\
\hline C & 1.175 & & & & & & & & & & & .197 \\
\hline C & 2.032 & 5.04 & & 1.606 & & & & & & & -0.2 & 7.056 \\
\hline $\mathrm{H}$ & 2.353 & 4.304 & 8.497 & 1.747 & & & 34 & & & & & 6.189 \\
\hline C & 2.452 & 6.375 & & & & & & -1.2 & & & & 7.052 \\
\hline $\mathrm{H}$ & 3.120 & 6.68 & & & & & & -1. & & & & 6.172 \\
\hline C & 2.008 & & & & & & & -2 & & & & 8.188 \\
\hline $\mathrm{H}$ & 2.310 & & & & & & & -3. & & & & 8.227 \\
\hline c & 160 & & & & & & & - & & & & .289 \\
\hline $\mathrm{H}$ & 0.778 & & & & & & & -2.4 & & & & 10.189 \\
\hline C & 0.671 & & & & & & & & & & & 8.340 \\
\hline $\mathrm{N}$ & -0.225 & 3.138 & 5.538 & -0.172 & & & 4.713 & & 9.249 & 4.660 & & 9.503 \\
\hline $\mathrm{N}$ & -0.513 & 1.838 & 5.464 & 0.533 & 1.932 & 5.246 & 5.001 & 3.245 & 9.323 & 5.021 & 3.151 & 9.541 \\
\hline C & 0.178 & 1.163 & 6.410 & 0.140 & 1.319 & 6.392 & 4.309 & 3.920 & 8.377 & 4.628 & 3.764 & 8.395 \\
\hline I & 0.066 & 0.087 & 6.513 & -0.357 & 0.265 & 6.553 & 4.422 & & 8.273 & 4.845 & 4.817 & 8.235 \\
\hline C & 0.960 & 2.074 & 7.123 & 0.504 & & 7.200 & 3.527 & 3.009 & 7.664 & & & 7.587 \\
\hline $\mathrm{H}$ & 1.640 & 1.860 & & 944 & & & & & & & & 6.608 \\
\hline B & -1.599 & 1.326 & 4.460 & -1.461 & 1.382 & 4.120 & 6.087 & & 10.327 & 5.949 & 01 & 10.667 \\
\hline $\mathrm{H}$ & -2.683 & 1.769 & 4.817 & -2.621 & 1.607 & & 7.171 & & 9.970 & & & 10.345 \\
\hline $\mathrm{H}$ & -1.562 & 0.108 & 4.453 & -1.250 & 0.186 & & 6.050 & 4.975 & 10.334 & 5.738 & 4.897 & 10.790 \\
\hline $\mathrm{N}$ & -1.265 & 1.841 & 3.028 & -1.154 & 2.084 & 2.759 & 5.753 & 3.242 & 11.759 & 5.642 & 2.999 & 12.028 \\
\hline $\mathrm{N}$ & -1.109 & 3.150 & 2.721 & -0.933 & 3.408 & 2.570 & 5.596 & 1.933 & 12.066 & 5.421 & 1.675 & 12.217 \\
\hline C & -1.000 & 3.237 & & -1.069 & 3.653 & & 5.488 & & & 5.557 & 1.430 & 13.528 \\
\hline $\mathrm{H}$ & -0.852 & 4.207 & 0.918 & -0.922 & 4.656 & 0.868 & 5.340 & 0.876 & & 5.410 & 0.427 & 13.919 \\
\hline C & -1.090 & 1.962 & 0.809 & -1.383 & 2.468 & & 5.578 & 3.122 & & 5.871 & 2.615 & 14.213 \\
\hline $\mathrm{H}$ & -1.043 & 1.698 & -0.244 & -1.553 & 2.338 & -0.491 & 5.531 & & & 6.041 & 2.745 & 15.279 \\
\hline C & -1.257 & & & & & & 5.745 & & 12.891 & & 3.584 & 13.217 \\
\hline & -1.384 & 0.026 & 1.942 & -1.648 & 0.436 & 1.524 & 5.872 & 5.057 & 12.846 & 6.136 & 4.647 & 13.263 \\
\hline
\end{tabular}

